\title{
Processo Markoviano de Decisão para a Alocação Dinâmica de Recursos e Controle Justo em Redes Ópticas WDM
}

\author{
Adriana N. F. da Rosa, Solon V. de Carvalho, Cynthia F. Leal, Carlos R. L. Francês, João C. W. A. Costa
}

\begin{abstract}
Resumo-Neste trabalho investigam-se políticas de alocação dinâmica de comprimentos de onda em redes ópticas WDM. Estuda-se um anel óptico unidirecional com $n$ nós e tráfego heterogêneo entre os diferentes pares origem - destino. Os intervalos entre chegadas e tempos de processamento das chamadas em cada nó são, por hipótese, independentes e exponencialmente distribuídos. Tem-se como objetivo, a determinação de uma política ótima que maximize o número médio de canais utilizados no anel. $O$ problema pode ser formulado como um processo markoviano de decisão (MDP) e um algoritmo de iteração de valores é utilizado para obtenção da política de alocação ótima. Resultados numéricos são apresentados.
\end{abstract}

Palavras-Chave-Redes Ópticas WDM (Wavelength Division Multiplexin), Alocação Dinâmica de Comprimento de Onda, Processo Markoviano de Decisão.

Abstract-This paper outlines policies for dynamic assignment of wavelengths in WDM optical networks. The study is based on a unidirectional optical ring with $n$ nodes with heterogeneous traffic between different source - destination pairs. The intervals between arrival and processing time of calls in each node are, by definition, independent and exponentially distributed. The purpose of work is the determination of an optimal policy that maximizes the average number of channels in use on the optical ring. The problem can be formulated as a Markov Decision Process (MDP) and an algorithm of iteration of values is used to obtain the optimal allocation policy. Numerical results are presented.

Keywords-Optical WDM Networks, Dynamic Wavelength Allocation, Markov Decision Process.

\section{INTRODUÇÃO}

Redes WDM (Wavelength Division Multiplexin) consistem em um conjunto de equipamentos e meios físicos que têm capacidade de otimizar o uso de redes de fibras ópticas. Em linhas gerais, esta tecnologia modula eletronicamente vários comprimentos de onda por sinais distintos e propaga - os simultaneamente na fibra óptica $[1$, $2,5,11]$. Ela possibilita que uma grande largura de banda

Adriana N. F. da Rosa, Cynthia F. Leal and João C. W A. Costa Laboratório de Eletromagnetismo Aplicado, Carlos R. L. Francês, Laboratório de Planejamento de Redes de Alto Desempenho, Universidade Federal do Pará, Belém, PA, E-mails: \{adrianan, cynthia, jweyl, rfrances\}@ufpa.br. Solon V. de Carvalho, Laboratório Associado de Computação e Matemática Aplicada, Instituto Nacional de Pesquisas Espaciais - INPE, São José dos Campos, SP, Brazil, E-mail: Solon@lac.inpe.br. Este Trabalho foi parcialmente financiado pelo CNPq e pela CAPES, projeto PROCAD número 0226050. de transmissão em fibra óptica seja dividida em múltiplos canais de comunicação os quais possuem bandas compatíveis com as velocidades de processamento eletrônico dos usuários finais [11].

Existe um grande interesse em redes ópticas WDM que utilizam roteamento e alocação do comprimento de onda entre os nós interconectados por fibra óptica. Estas redes transportam dados entre as estações de acesso no domínio óptico, sem qualquer conversão eletrônica intermediária. Para que seja possível enviar os dados de um nó para outro, é necessário estabelecer uma conexão na camada óptica, semelhante a um circuito de rede comutada. Isso pode ser realizado através da determinação de uma via fim-a-fim (linghpath) entre dois nós e a atribuição de um comprimento de onda disponível em todos os enlaces ao longo do caminho estabelecido. A largura de banda em toda a via fim-a-fim é reservada para uma dada conexão até que a mesma seja encerrada. Quando uma conexão é encerrada, o comprimento de onda associado torna-se disponível em todos os links ao longo do percurso [11].

$\mathrm{Na}$ ausência da conversão do comprimento de onda, é necessário que o linghpath ocupe o mesmo comprimento de onda em todos os enlaces de fibra utilizados. Esta exigência é referida como Wavelength Continuity Constraint. Tal problema pode ser relaxado pelo uso de conversores de comprimento de onda (WC's) em nós intermediários [12].

A determinação de rota e de comprimento de onda constitui-se em um problema complexo chamado de problema RWA (Routing and Wavelength Assignment) e consiste uma importante questão em redes WDM [7]. Em geral, costuma-se dividi-lo em dois subproblemas: $i$ ) Problema de atribuição de comprimento de onda e ii) Problema de roteamento.

Algoritmos para a solução do problema RWA têm sido propostos $[3,6,7,8]$ de modo a garantir o estabelecimento de rotas e a designação do comprimento de onda para vias fim-a-fim dentro de uma rede óptica WDM. Em geral, eles diferem nas suposições sobre o padrão de tráfego, disponibilidade de comprimento de onda e objetivos desejáveis. Os tráfegos pressupostos geralmente caem em uma das duas categorias: estáticos ou dinâmicos. 
Nos modelos RWA estáticos admite-se que a demanda é fixa e conhecida, ou seja, todos os linghpath que serão estabelecidas são conhecidos antecipadamente. O objetivo é tipicamente atender a demanda e ao mesmo tempo minimizar o número de comprimentos de onda utilizados em todos os links. Diferente do modelo estático, no modelo dinâmico admite-se que as requisições de vias fim-a-fim, entre os pares origem-destino, chegam randomicamente, e possuem um tempo de encerramento aleatório. O objetivo principal, neste caso, consiste em minimizar a probabilidade de bloqueio, ou minimizar o total (de forma ponderada) do número de conexões bloqueadas durante um dado período de tempo.

Pesquisas mostram que a topologia da rede, a utilização de conversores ópticos e a utilização de algoritmos adequados para o problema RWA constituem a base para melhorar o desempenho de redes ópticas WDM.

A implementação de soluções para o problema RWA que permitam determinar rotas em uma rede óptica inteligente, otimizando os recursos e minimizando a probabilidade de bloqueio de requisições de vias fim-a-fim, torna-se fundamental nesta nova concepção [4, 12]. Com uma boa solução, mais clientes podem ser atendidos pelo sistema, e menos clientes necessitam ser rejeitados durante períodos de congestionamento. Temos assim, a minimização do bloqueio de conexões e, por conseguinte, a degradação do desempenho da rede.

Dentre os muitos aspectos de redes ópticas WDM e do problema RWA, destaca-se, neste trabalho, o problema da alocação de comprimento de onda em redes ópticas WDM, provendo alocação dinâmica e controle justo de recursos. O problema pode ser formulado como um Processo Markoviano de Decisão (MDP) [9], cujo objetivo consiste na determinação de uma política ótima para a atribuição de comprimento de onda a fim minimizar a probabilidade de bloqueio em cada nó do anel e maximizar o número médio de canais utilizados por três diferentes classes de usuários.

O trabalho é organizado da seguinte forma. Na seção II temos a definição do problema. Na seção III, por sua vez, a formulação do mesmo como um processo markoviano de decisão (MDP) para um anel óptico unidirecional com $n$ nós. Na seção IV, são apresentados resultados numéricos. As considerações finais e perspectivas de trabalhos futuros são apresentadas na seção V.

\section{ALOCAÇÃO DINÂMICA DE COMPRIMENTO DE ONDA PARA DIFERENTES CLASSES DE USUÁRIOS}

Política de alocação do comprimento de onda é um problema particular relacionado à política de alocação de recursos. Em geral, estratégias para a alocação do comprimento de onda estão incorporadas em algoritmos heurísticos tais como complete sharing (CS) e complete partitioning (CP) [6, 7, 8]. Na política CS nenhum comprimento de onda é reservado para qualquer classe e uma chamada só será aceita se no mínimo um comprimento de onda estiver disponível em todo o caminho a ser utilizado pela chamada. Esta abordagem apresenta uma alta utilização da rede global e será subótima se diferentes classes de usuários proverem "retornos" diferentes para o mesmo grau de serviço. A política $\mathrm{CP}$, por sua vez, atribui para cada classe de chamada um número constante de comprimento de onda, que não pode ser utilizado por chamadas de outras classes. Desta forma, ela suporta serviços diferenciados e controle de probabilidade de bloqueio das classes base. Entretanto, a política $\mathrm{CP}$ não maximiza a utilização de todos os recursos disponíveis [8].

Para melhorar o desempenho do sistema em um ambiente dinâmico, seria essencial atribuir certo número de comprimentos de onda para cada classe em função do número de clientes das diferentes classes [8]. Neste contexto, investiga - se uma política de alocação dinâmica do comprimento de onda, que consiste na determinação da aceitação ou não das chamadas de cada classe que chegam a um dado nó do anel, tendo em conta o estado atual do sistema, com o objetivo de maximizar o número médio de canais utilizados pelas classes.

Considera - se um anel óptico unidirecional com $n$ nós como mostrado na Figura 1. Cada enlace possui o mesmo número de comprimentos de onda $W$. O tráfego é gerado (ou oferecido à rede) na forma de chamadas. Uma chamada é essencialmente uma requisição feita por um dado nó para que seja reservado um conjunto fixo de recursos para uso exclusivo do fluxo de informações associado à chamada.

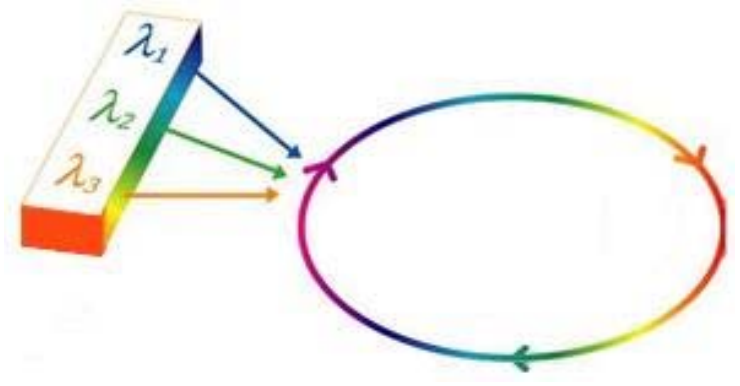

Fig 1 - Anel óptico unidirecional

O tráfego na rede é heterogêneo, ou seja, cada nó possui probabilidades diferentes de gerar tráfego. Além disso, cada chamada originada na rede destina - se apenas a um único nó destino, não sendo permitido, portanto, o multicasting de chamadas.

As chamadas entre dois nós quaisquer chegam ao anel de acordo com processos de Poisson independentes, cada um com taxa $\lambda$. Os tempos de duração das chamadas são independentes e exponencialmente distribuídos com taxa $\mu$. Uma chamada que chega à rede será alocada e o seu serviço começará imediatamente se os recursos estiverem disponíveis. Caso contrário, considera- se que a chamada é perdida. Não há mecanismos de espera nestas redes. 
Cada nó é modelado como um Processo Markoviano de Decisão. No modelo de cada nó considera-se que cada chamada que chega ao nó é classificada em uma das três classes de serviço. A classe 1 corresponde às chamadas que se destinam ao nó; a classe 2 corresponde às chamadas que passam pelo nó; a classe 3 , por sua vez, consiste nas chamadas que partem do nó em questão. No instante de chegada de uma nova chamada, em função da quantidade de chamadas já em transmissão pelo nó e da classe que chegou, deve-se decidir sobre sua aceitação de forma a otimizar o uso dos recursos disponíveis no nó.

Na Figura 2 temos $\lambda_{\mathrm{i}}, i \in\{1,2,3\}$ que corresponde à taxa de chegada de chamadas da classe $i, i \in\{1,2,3\}$, em um dado nó do anel, enquanto que $\lambda^{\prime}{ }_{\mathrm{i}}, \mathrm{i} \in\{1,2,3\}$ consiste na taxa de chegadas que efetivamente entram no nó de observação, isto é, que não são bloqueadas no nó.

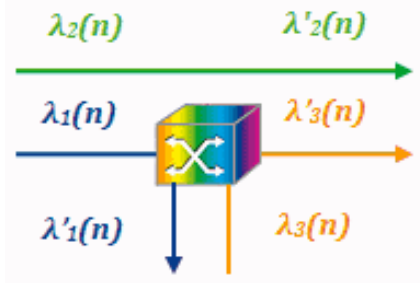

Fig 2 - Modelo de um nó do anel

Supondo conhecidas as probabilidades de bloqueio das chamadas de cada classe em cada nó, e supondo, por aproximação, que o comportamento dos nós é independente, é possível calcular a probabilidade de bloqueio de cada chamada no anel em função dos seus nós de origem e destino. Por exemplo, dado um anel unidirecional com 4 enlaces, uma chamada originada no nó 1 e destinada ao nó 3 , será transmitida se não for bloqueada no nó 1 (neste nó a chamada é da classe 3), nem no nó 2 (classe 2) e nem no nó 3 (classe 1 ). Assim a probabilidade de bloqueio neste caso é dada por:

$$
1-[1-B 3(1)][1-B 2(2)][1-B 1(3)
$$

O modelo proposto para os nós será detalhado na seção seguinte. Sua resolução para cada nó fornece a política de controle de aceitação de novas chamadas e a probabilidade de bloqueio de uma chamada pertencente a cada uma das três classes consideradas. Estas probabilidades são B1(i), $B 2(i)$ e $B 3(i)$ para as classes 1,2 e 3 respectivamente.

O modelo proposto para o anel agrega e compatibiliza iterativamente os resultados obtidos pelos modelos individuais dos nós. Considerando ainda o modelo do anel óptico unidirecional com 4 enlaces, para cada nó, definese uma função $v(n, s)$ que, a partir de um nó de referência $n(0 \leq n \leq 3)$ e de uma variação de posição $s(-3 \leq s \leq 3)$, retorna o índice do nó no anel em tal posição. Esta função é dada por:

$$
v(n, s)= \begin{cases}n+s & \text { se } 1 \leq n+s \leq n \\ n+s-4 & \text { se } n+s>4 \\ n+s+4 & \text { se } n+s<4\end{cases}
$$

E será usada no Algoritmo 1 mostrado a seguir que corresponde à resolução do modelo proposto para o anel.

\section{Algoritmo 1}

$$
\begin{aligned}
& \text { Inicializar } B_{I}(i) \leftarrow 0, B_{2}(i) \leftarrow 0 \text { e } B_{3}(i) \leftarrow 0 \text { para } i=1,2,3 \text { e } 4 \\
& \text { Repetir } \\
& \text { ErroIteraçãoŁo } \\
& \text { Para i de } 1 \text { até } 4 \text { fazer: } \\
& \lambda_{1} \leftarrow \lambda\left\{\left[1-B_{3}(v(i,-3)]\left[1-B_{2}(v(i,-2)]\left[1-B_{2}(v(i,-1)]+\right.\right.\right.\right. \\
& {\left[1-B_{3}(v(i,-2)]\left[1-B_{2}(v(i,-1)]+\right.\right.} \\
& {\left[1-B_{3}(v(i,-1)]\right. \text { \} }} \\
& \lambda_{2} \leftarrow \lambda\left\{\left[1-B_{3}(v(i,-2)]\left[1-B_{2}(v(i,-1)]\left[1-B_{1}(v(i,+1)]\right.\right.\right.\right. \\
& +\left[1-B_{3}(v(i,-1)]\left[1-B_{1}(v(i,+1)]+\right.\right. \\
& {\left[1-B_{3}(v(i,-1)]\left[1-B_{2}(v(i,+1)]\left[1-B_{1}(v(i,\right.\right.\right.} \\
& +2)]\} \\
& \lambda_{3} \leftarrow \lambda\left\{\left[1-B_{1}(v(i,+1)]+\right.\right. \\
& {\left[1-B_{2}(v(i,+1)]\left[1-B_{1}(v(i,+2)]+\right.\right.} \\
& {\left[1-B_{2}(v(i,+1)]\left[1-B_{2}(v(i,-2)]\left[1-B_{1}(v(i,\right.\right.\right.} \\
& -1)] \quad \text { \} } \\
& \operatorname{Erro}(i) \leftarrow \max \left\{\begin{array}{c}
\left|B_{1}(i)-C_{1}\right|,\left|B_{2}(i)-C_{2}\right|, \\
\left|B_{3}(i)-C_{3}\right|
\end{array}\right\} \\
& \text { Se ErroIteração }<\text { Erro(i) Fazer ErroIteração } \leftarrow \operatorname{Erro}(i) \\
& \text { Fimse } \\
& \text { FimPara }
\end{aligned}
$$

\section{POLÍTICA ÓTIMA BASEADA EM UM PROCESSO MARKOVIANO DE DECISÃO}

Nesta seção, descreve-se a modelagem markoviana proposta para o problema descrito na seção anterior. Cada estado do sistema é definido pelo conjunto de valores $\left(n_{1}\right.$, $\left.n_{2}, n_{3}, e v\right)$, onde $e v$ corresponde ao último evento ocorrido no sistema, podendo assumir os valores $a_{i}$ ou $s_{i}$, se a última ocorrência de evento for a chegada ou o término de processamento de uma chamada da classe $i \in\{1,2,3\}$ respectivamente. Ainda em relação a cada estado, $n_{i}, i \in$ $\{1,2,3\}$, corresponde ao número de comprimentos de ondas utilizados pela classe $i$, imediatamente antes da ocorrência do evento $e v$. Desta forma, o espaço de estados $S$ do modelo proposto considerando um anel óptico unidirecional com tráfego heterogêneo, onde os nós possuem probabilidades diferentes de gerar de tráfego, é:

$$
\begin{aligned}
& S=\left\{\left(n_{1}, n_{2}, n_{3}, \text { ev }\right)\right. \mid n_{1} \geq 0, n_{2} \geq 0, n_{3} \geq 0, e \in\left\{a_{1}, a_{2}, a_{3}, s_{1}, s_{2}, s_{3}\right\}, \\
& n_{1}+n_{2} \leq W, n_{2}+n_{3} \leq W, \\
& \text { se } n_{1}=0 \text { então ev } \neq s_{1}, \\
& \text { se } n_{2}=0 \text { então ev } \neq s_{2}, \\
&\text { se } \left.n_{3}=0 \text { então ev } \neq s_{3}\right\}
\end{aligned}
$$

Por hipótese, o sistema é observado continuamente no tempo. A cada chegada de uma nova chamada, deve-se decidir sobre sua aceitação ou rejeição ( $A$ ou $R$ ). Uma 
nova chamada só poderá ser aceita quando houver comprimentos de onda para alocá-la. A cada término de processamento de chamada, esta sai do sistema e nenhuma decisão é tomada, ou seja, deve-se adotar a ação $N$ (nenhuma ação). Assim para cada estado $s=\left(n_{1}, n_{2}, n_{3}\right.$, $e v) \in \mathrm{S}$, as ações possíveis $A(s)$, em cada nó do anel, são como se segue.

$$
A(s)=\left\{\begin{array}{l}
\{A C C, R E J\} \text { se }\left\{\begin{array}{l}
\left(e=a_{1} \wedge n_{1}+n_{2} \leq W\right) \vee \\
\left(e=a_{2} \wedge n_{1}+n_{2} \leq W \wedge n_{2}+n_{3} \leq W\right) \vee \\
\left(e=a_{3} \wedge n_{2}+n_{3} \leq W\right)
\end{array}\right. \\
\{R E J\} \quad \text { se }\left\{\begin{array}{l}
\left(e=a_{1} \wedge n_{1}+n_{2}=W\right) \vee \\
\left(e=a_{2} \wedge\left(n_{1}+n_{2}=W \vee n_{2}+n_{3}=W\right)\right) \vee \\
\left(e=a_{3} \wedge n_{2}+n_{3}=W\right)
\end{array}\right. \\
\left\{\begin{array}{l}
\text { se } e=s_{1} \vee e=s_{2} \vee e=s_{3} \\
\text { NOA }
\end{array}\right.
\end{array}\right.
$$

O comportamento dinâmico do sistema é definido pela observação contínua do estado corrente do sistema $s=\left(n_{1}\right.$, $n_{2}, n_{3}$, ev $\in S$, e pela ação adotada $a \in A(s)$ cada vez que este estado se altera, ou seja, quando ocorre um novo evento. Admite-se que a decisão é tomada imediatamente após a observação da ocorrência do evento.

Se o estado $s=\left(n_{1}, n_{2}, n_{3}, e v\right) \in \mathrm{S}$ é observado e uma ação $a \in A(s)$ é escolhida, o sistema deve passar imediatamente a um "estado" reagido $s_{r}$ apresentado na Tabela I, e aguardar a ocorrência do próximo evento, quando ocorrerá a próxima transição. Se o último evento em $s \in S$ é a chegada de uma chamada e é tomada a decisão de aceitá-la, o sistema imediatamente muda para um "estado" reagido $s_{r}$ em que a nova chamada é incorporada ao sistema. Se o último evento é o término de uma chamada, a única ação possível é não fazer nada e a chamada deve ser imediatamente retirada do sistema. As condições do estado $\mathrm{s} \in \mathrm{S}$, a ação tomada $\mathrm{a} \in \mathrm{A}(\mathrm{s})$ e o estado reagido $s_{r}$, são apresentados na Tabela I.

Na Tabela II, por sua vez, apresentam - se o próximo estado e a taxa de cada transição, em função do estado reagido a que chega o sistema e do próximo evento a ocorrer. Note que as transições possíveis são combinações das linhas das Tabelas I e II.

TABELA I

ESTADO REAGIDO.

\begin{tabular}{|c|c|c|}
\hline Condição & Ação & $\begin{array}{c}\text { Estado Reagido } \\
\mathbf{s}_{r}=\left(r_{1}, r_{2}, r_{3}\right)\end{array}$ \\
\hline ev $=a_{1} \wedge n_{1}+n_{2}<W$ & $A$ & $\left(n_{1}+1, n_{2}, n_{3}\right)$ \\
\hline$e v=a_{2} \wedge n_{1}+n_{2}<W$ & $A$ & $\left(n_{1}, n_{2}+1, n_{3}\right)$ \\
\hline
\end{tabular}

\begin{tabular}{|l|l|l|}
\hline$\wedge \mathrm{n}_{2}+\mathrm{n}_{3}<\mathrm{W}$ & & \\
\hline $\mathrm{ev}=\mathrm{a}_{3} \wedge \mathrm{n}_{2}+\mathrm{n}_{3}<\mathrm{W}$ & $\mathrm{A}$ & $\left(\mathrm{n}_{1}, \mathrm{n}_{2}, \mathrm{n}_{3}+1\right)$ \\
\hline $\mathrm{ev}=\mathrm{s}_{1}\left(\mathrm{n}_{1}>0\right)$ & $\mathrm{N}$ & $\left(\mathrm{n}_{1}-1, \mathrm{n}_{2}, \mathrm{n}_{3}\right)$ \\
\hline $\mathrm{ev}=\mathrm{s}_{2}\left(\mathrm{n}_{2}>0\right)$ & $\mathrm{N}$ & $\left(\mathrm{n}_{1}, \mathrm{n}_{2}-\mathrm{n}_{3}\right)$ \\
\hline ev $\left.=\mathrm{s}_{3} \mathrm{n}_{3}>0\right)$ & $\mathrm{N}$ & $\left(\mathrm{n}_{1}, \mathrm{n}_{2}, \mathrm{n}_{3}-1\right)$ \\
\hline caso contrário & $\mathrm{R}$ & $\left(\mathrm{n}_{1}, \mathrm{n}_{2}, \mathrm{n}_{3}\right)$ \\
\hline
\end{tabular}

TABELA II

PRÓXIMO ESTADO E TRANSIÇÃO ASSOCIADA.

\begin{tabular}{|c|c|c|}
\hline $\begin{array}{c}\text { Próximo } \\
\text { Evento }\end{array}$ & Próximo Estado & $\begin{array}{c}\text { Taxa de } \\
\text { Transição }\end{array}$ \\
\hline$a_{1}$ & $\left(r_{1}, r_{2}, r_{3}, a_{1}\right)$ & $\lambda_{1}$ \\
\hline$a_{2}$ & $\left(r_{1}, r_{2}, r_{3}, a_{2}\right)$ & $\lambda_{2}$ \\
\hline$a_{3}$ & $\left(r_{1}, r_{2}, r_{3}, a_{3}\right)$ & $\lambda_{3}$ \\
\hline $\mathbf{s}_{1}$ & $\left(r_{1}, r_{2}, r_{3}, s_{1}\right)$ & $r_{1} \mu_{1}$ \\
\hline $\mathbf{s}_{2}$ & $\left(r_{1}, r_{2}, r_{3}, s_{2}\right)$ & $r_{2} \mu_{2}$ \\
\hline $\mathbf{s}_{3}$ & $\left(r_{1}, r_{2}, r_{3}, s_{3}\right)$ & $r_{3} \mu_{3}$ \\
\hline
\end{tabular}

Para finalizar a modelagem do Processo Markoviano de Decisão (MDP), é necessário definir uma função de retorno $R(s, a)$, que corresponde ao retorno total esperado do sistema até o próximo instante de observação, dado que sistema é observado no estado $s=\left(n_{1}, n_{2}, n_{3}, e v\right) \in S$, e a ação tomada $a \in A(s)$ é escolhida.

No modelo, o retorno corresponde ao número de chamadas sendo transmitidas no estado corrente, eventualmente ponderados por pesos $r_{1}, r_{2}$ e $r_{3}$ atribuídos à ocupação de canais por cada uma das classes $i, i \in\{1,2$, $3\}$. Como imediatamente após a escolha da ação, o sistema passa ao "estado" reagido $s_{r}=\left(t_{1}, t_{2}, t_{3}, e v_{r}\right)$ mostrado na Tabela II, é em função do estado reagido que se deve obter o retorno $R(s, a)$ da seguinte maneira:

$$
R(s, a)=\left(r_{1} t_{1}+r 2 t_{2}+r_{3} t_{3}\right) \tau(s, a)
$$

Onde $\tau(s, a)$ corresponde ao tempo esperado até o próximo instante de decisão, que é dado por $\tau(s, a)=\sum_{s \neq s^{\prime}} \Lambda_{s s^{\prime}}(a)$. $\Lambda_{s s}$ (a) consiste na taxa de transição do estado $\mathrm{s} \in \mathrm{S}$ para o estado $s^{\prime} \in S$, quando a ação $a \in A(s)$ é escolhida.

Por fim, tem - se que o throughput de chamadas no estado corrente ponderado por pesos $r_{1}, r_{2}$ e $r_{3}$ atribuídos a cada uma das classes é dado por:

$$
T(s, a)=\left(r_{1} t_{1} \mu_{1}+r_{2} t_{2} \mu_{2}+r_{3} t_{3} \mu_{3}\right) \tau(s, a)
$$

\section{RESULTADOS NUMÉRICOS}

Para testar o modelo supõem-se o seguinte conjunto de dados. Todos o enlaces possuem o mesmo número de canais disponíveis, dado por 8 comprimentos de onda. As taxas de chegada de chamadas $\lambda_{i}, i \in\{1,2,3\}$, em um dado nó do anel, são diferenciadas e seguem uma distribuição exponencial. A taxa de processamento das chamadas é dada por $\mu_{1}=\mu_{2}=\mu_{3}=1$. Considera-se que todas as classes de usuários possuem o mesmo peso, isto é, $r_{i}=1, i \in\{1,2,3\}$.

No modelo markoviano de decisão proposto, admite-se um anel óptico unidirecional com $n$ nós. Para fins de simulação, considerou-se 5 nós do anel. 
Para os dados considerados, a política ótima obtida determina nunca rejeitar chamadas do tipo 1 ou $3 \mathrm{e}$ rejeita as chamadas do tipo 2 quando o número destas chamadas for maior ou igual à $\mathrm{N}_{2}{ }_{2}$.

As Figuras 3 e 4 ilustram o nível máximo de aceitação das chamadas para as três classes de usuários, com base na política ótima obtida.

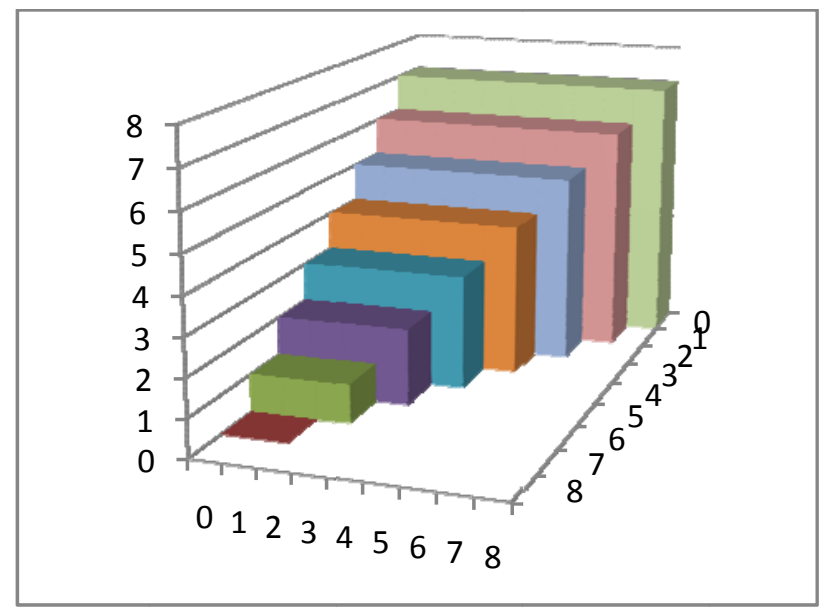

Fig 3 - Nível máximo de aceitação das chamadas das classes 1 e 3

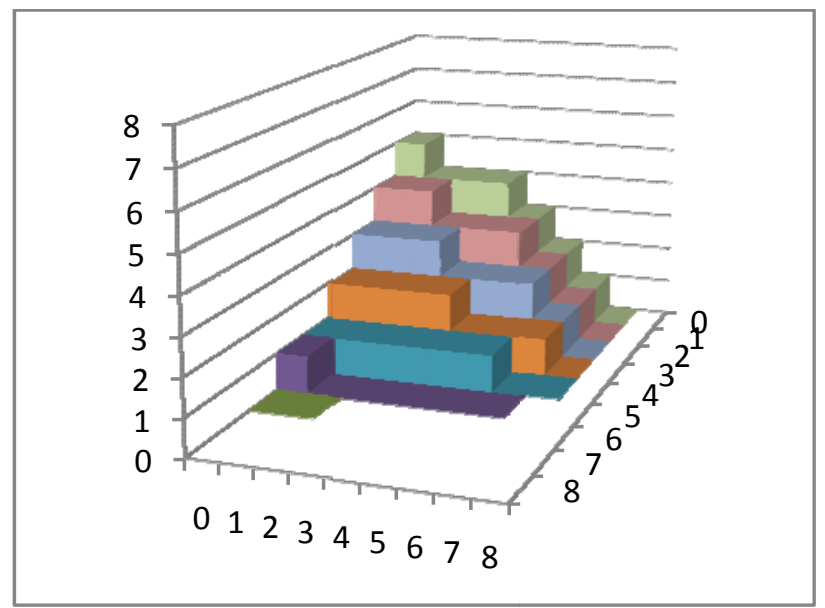

Fig 4 - Nível máximo de aceitação das chamadas da classe 2

Pode-se observar que quanto maior a carga do sistema (taxa de chegada de chamadas) maior será a probabilidade de bloqueio em cada classe, sendo que o bloqueio das chamadas da classe 2 é maior do que o bloqueio de chamadas nas outras classes. Este resultado indica que a política está dando prioridade para as classes que entram e saem do nó, praticamente não permitindo a chegada de chamadas que simplesmente passam pelo nó, ou seja, chamadas que devem ser retransmitidas para os nós seguintes.
Na Figura 5 temos as taxas de chegada de chamadas, em cada nó do anel, com base na política ótima, para as três diferentes classes de usuário.

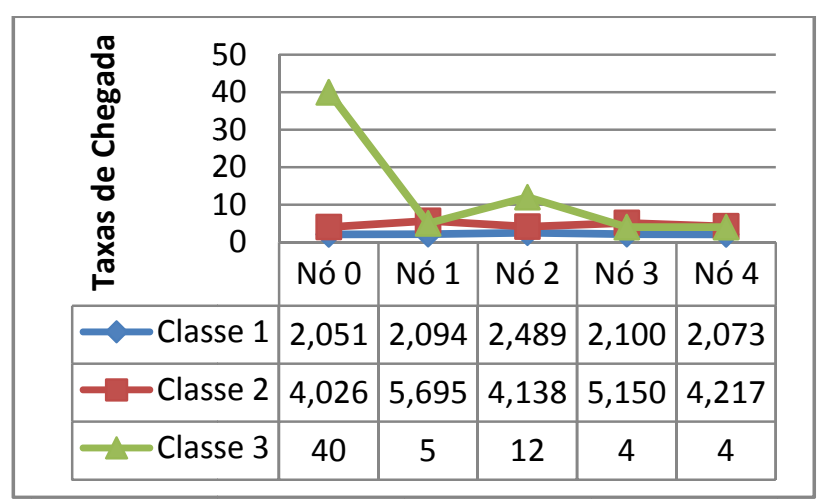

Fig 5 - Taxa de chegada de chamada para as três classes de usuários

Com base na política ótima, temos ainda a probabilidade de bloqueio e o throughput para as três classes de usuário em cada nó do anel, como se segue nas Figuras 6 e 7, respectivamente.

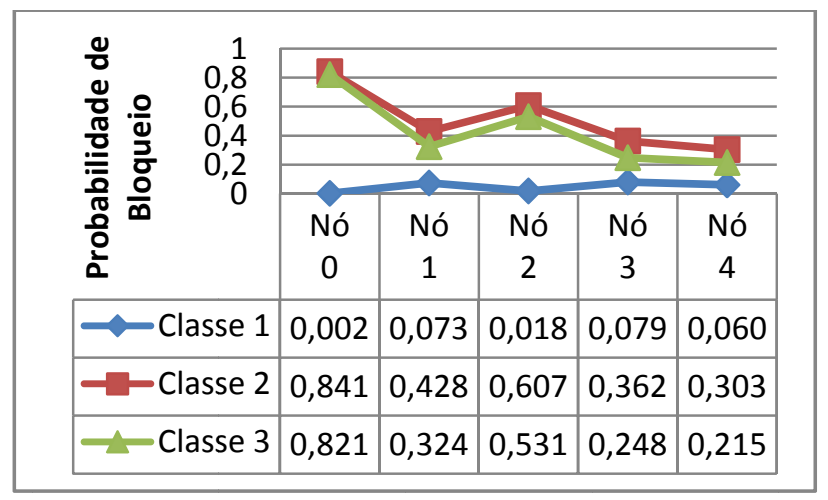

Fig 6 - Probabilidade de bloqueio para as três classes de usuários

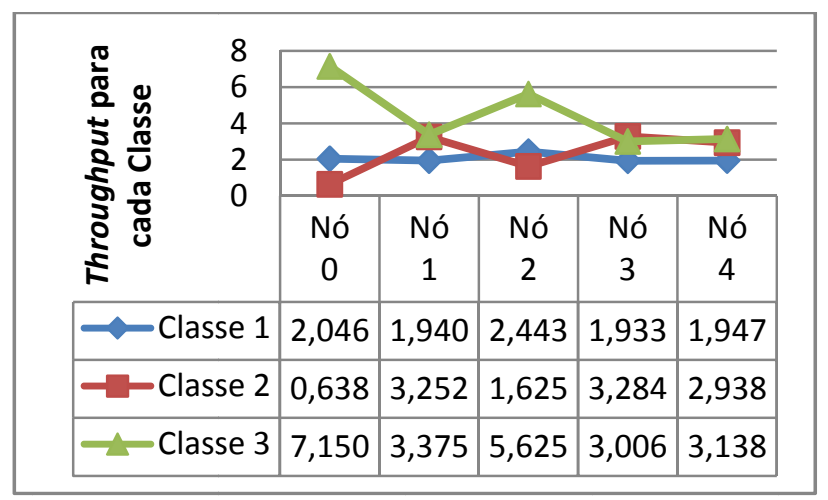

Fig 7 - Throughput para as três classes de usuários

NaTabela III temos a probabilidade de bloqueio e o throughput em cada via fim-a-fim (linghpath) estabelecida. Observa - se que as que chamadas percorrem um maior 
número de enlace possuem maior probabilidade de bloqueio, haja vista que utilizarão por maior tempo os recursos da rede.

\section{TABELA III}

PROBABILIDADE DE BLOQUEIO E THROUGHPUT EM CADA CONEXÃO

\begin{tabular}{|c|c|c|}
\hline $\begin{array}{l}\text { Via fim- } \\
\text { a-fim }\end{array}$ & $\begin{array}{l}\text { Probabilidade } \\
\text { de Bloqueio }\end{array}$ & Throughput \\
\hline$(0,1)$ & 0.834338 & 1.65662 \\
\hline$(0,2)$ & 0.89981 & 1.0019 \\
\hline$(0,3)$ & 0.963075 & 0.369247 \\
\hline$(0,4)$ & 0.975968 & 0.240319 \\
\hline$(1,0)$ & 0.88243 & 0.11757 \\
\hline$(1,2)$ & 0.337482 & 1.32504 \\
\hline$(1,3)$ & 0.755831 & 0.244169 \\
\hline$(1,4)$ & 0.841086 & 0.158914 \\
\hline$(2,0)$ & 0.792208 & 0.623376 \\
\hline$(2,1)$ & 0.969392 & 0.0918252 \\
\hline$(2,3)$ & 0.568458 & 1.29463 \\
\hline$(2,4)$ & 0.719137 & 0.84259 \\
\hline$(3,0)$ & 0.477612 & 0.522388 \\
\hline$(3,1)$ & 0.923051 & 0.0769493 \\
\hline$(3,2)$ & 0.953462 & 0.0465378 \\
\hline$(3,4)$ & 0.293912 & 0.706088 \\
\hline$(4,0)$ & 0.217108 & 0.782892 \\
\hline$(4,1)$ & 0.884678 & 0.115322 \\
\hline$(4,2)$ & 0.930255 & 0.0697453 \\
\hline$(4,3)$ & 0.974296 & 0.0257044 \\
\hline
\end{tabular}

Por fim, tem-se a análise do índice de "justiça" da política ótima proposta através da resolução da seguinte equação [10].

$$
\begin{gathered}
f(\mathbf{x})=\frac{\left[\sum_{i=1}^{n} x_{i}\right]^{2}}{n \sum_{i=1}^{n} x_{i}^{2}} \quad x_{i}=0 \\
x_{i}=\frac{\lambda_{i j}^{o}}{\lambda_{i j}^{i}}
\end{gathered}
$$

Com base na mesma tem-se que índice de "justiça" para a rede é de 0.502743 . Já o throughput para o anel unidirecional com 5 enlaces é de 10.3118 .

\section{CONCLUSÕES}

O trabalho propôs um modelo de decisão markoviano para um anel óptico unidirecional com tráfego heterogêneo, onde todos os nós da rede possuem diferentes probabilidade de geração de tráfego. Obtiveram-se, políticas ótimas de alocação de comprimento de onda a fim de maximizar o número médio de canais utilizados pelas três diferentes classe de serviço em cada nó. Observou-se, que mesmo admitindo classes de tráfico com o mesmo peso, para os dados numéricos considerados neste trabalho, a política ótima priorizou as chamadas que entram e saiam do nó, em detrimento das que passam pelo nó.

Para trabalhos futuros, pretende-se utilizar outras funções objetivos, incorporando o conceito de políticas de controle "justas" já no processo de otimização. Pretendese também utilizar este tipo de modelagem para a análise de anéis ópticos bidirecionais, com tráfego heterogêneo, onde cada nó possui além de seu próprio tráfego, que é função da posição e dos serviços prestados pelo nó na rede, um modelo de roteamento inteligente.

\section{REFERÊNCIAS}

[1]Ayan, B. Kireeti, K. Yakov, R. "Generalized Multiprotocol Label Switching: Na Overview of Routing and Management Enhancements". IEEE Communications Magazine, Janeiro/2001.

[2]Ayan, B. Optical Communication Networks. McGraw - Hill, New York, 1997. [3]Biswanath, M. WDM optical communication networks: progress and challenges, IEEE J. Sel. Areas Commun. 18 (10) (2000).

[4]Dhritiman, B. Biswanath, M.. "Pratical approaches for routing and wavelength wavelength assignment in large all-optical wavelength-routed networks." IEEE Journal on Selected Areas in Communications, 14 (5): 903-908, june. (1998).

[5]Hui, Z. Jason, P. J. Biswanath M. - "A review of routing and wavelength assignment approaches for wavelength-routed optical WDM networks", Optical Networks Magazine, vol. 1, no. 1, Jan. 2000, pp. 47-60.

[6] Kayvan, M. J'erôme, T. Ioannis, L. "A Markov Decision Process Model for Dynamic Wavelength Allocation in WDM networks", IEEE GLOBECOM, San Francisco, CA, Dec. 2003, pp. 2590-2594.

[7]Kayvan, M. J'erôme, T. Ioannis, L. "Multithreshold Wavelength Allocation Policy for Fairness Control in WDM Ring Networks", Carleton Univ., Ottawa, ON, Canada, Tech. Rep., SCE-04-13, 2004.

[8] Kayvan, M. J'erôme, T. Ioannis, L. "Optimal Resource Allocation and Fairness Control in All-Optical WDM Networks", IEEE Journal on Selected Areas in Communications, vol. 23, no. 8, august 2005.

[9] Puterman, M. L. Markov Decision Processes. New York:Wiley, 1994.

[10]Rain J. "A Quantitative Measure Of Fairness And Discrimination For Resource Allocation In Shared Computer System"s, Technical Report DEC-TR301, 1984.

[11] Shinya I. "A Study on Flexible, Reliable, and Scalable Wavelength-Routed Optical Networks". PhD Thesis. Department of Information Networking, Graduate School of Information Science and Technology, Osaka University. February 2007. [12] Zhenssheng, Z. James, F, Guo, D. Zhang, L.; "Linghpath Routing for Intelligent Optical Networks". IEEE Networks, Agosto/2001. 